\title{
Enhancement of the Bond Duration-Convexity Approximation
}

\author{
Souad Lajili \\ Université Paris Est Créteil, IRG, Institut de Recherche en Gestion \\ Place de la Porte des Champs, 4 route de Choisy, 94010 Créteil, France \\ E-mail: souad.lajili-jarjir@u-pec.fr
}

Yves Rakotondratsimba

ECE Paris, Graduate School of Engineering, lab, ENSRF

37 quai de Grenelle CS71520 75725 Paris 15, France

E-mail: w_yrakoto@yahoo.com

Received: August 29, 2011

Accepted: January 4, $2012 \quad$ Published: March 1, 2012

doi:10.5539/ijef.v4n3p115

URL: http://dx.doi.org/10.5539/ijef.v4n3p115

\begin{abstract}
Hedging bond positions under the assumption of a parallel shift of the interest rate curve is well-known and used for a long date in finance. The approximation duration-convexity introduced by L. Fisher and R. Weil is the corresponding main tool. However this last is inaccurately formulated since: the time-passage is neglected, the shift size is assumed to be infinitesimal and the error approximation is out of the control. Our main purpose here is to present how to enhance this classical approximation such that these simultaneous inconveniences may be overcome. Not only our modified approximation leads to a perfect fit of the bond change, but a shift of any arbitrary size is also allowed and a deterministic and explicit estimates of the approximation error becomes available. Though the parallel shift of the interest rate curve is a strong and unrealistic assumption, it remains a standard reference among practitioners and academics. The analysis performed here finds a valuable implication in stress-testing, hedging and managing interest rate risks under more realistic models.
\end{abstract}

Keywords: Bonds, Duration, Convexity, Interest rate risks

JEL Classification: G11, G12.

\section{Introduction}

The 2007-08 crisis has put in surface malfunctioning of some glorified financial models and misuses of the existing models by various people (financial engineering, quants, risk managers, . . .), see Triana (2010). Revisiting and improving the foundations of some financial and technical models are among the issues considered by leading academic searchers and supervisory organizations, see Derman (2010) and Jarrow (2010). As a little contribution on this effort of clarification, we consider here the classical duration-convexity approximation initiated by L. Fisher and R. Weil (1971), which is useful when hedging a bond position with respect to a parallel shift of the interest rate curve. Though such an approximation is well-known and becomes among the standard tools in interest risk management, the situation is really inaccurately formulated such that the obtained result is very questionable. Among the inconveniences which may be raised are the following: 1) the time-passage is neglected; 2) the shift is assumed to be infinitesimal but the sense of this last is not clear; 3) the approximation error remains unknown.

Our main purpose here is to present how this classical Fisher-Weil bond duration-convexity approximation can be enhanced, such that we are able to solve simultaneously all of these three inconveniences. The parallel shift assumption underlying the duration-convexity approximation is well-known to be a too strong, not realistic assumption and not consistent with the no-arbitrage assumption, which is the basic assumption for valuation, see for instance Hegde \& Nunn (1988) and Nawalka \& Sotto (2009). However there are at least four reasons why the Fisher-Weil approach still deserves our consideration here. Indeed

- Parallel shift assumption is historically well-known and used as a benchmark approach of interest rate risk measure among practitioners and academics Our contribution here is to provide a consistent analysis and enhancement for this standard. 
- A parallel positive shift (as 100 basis points) remains also a standard and easily tractable approach in the perspective of stress-testing. The result we obtain in this paper enables the user to consider any large shifts lying inside a given range. This is particularly useful to grasp turmoil situations as we are faced frequently since the 2007 financial crisis. Moreover any non-parallel shift of the interest rate term structure may be seen as including in some range corresponding to suitable high and low parallel shifts.

- A correct approach and understanding of the situation related to the parallel shift is a main step to provide a good risk management analysis for the case where the interest rate curve term structure moves in a non-parallel fashion. We will develop in a next project that the ideas underlying this work lead to new and practical results related to bond risk management in the setting of a one-factor model of short interest rate.

- Though it is restrictive, considering a parallel shift of the interest rate has also the advantage to avoid the consideration of the shift distribution nature and consequently gives a fast and clear vision of the core of the interest risk issue.

The parallel shift hypothesis is not consistent with the no-arbitrage assumption. Though alternative consistent models do exist, each of them remains a metaphor of the reality, see Derman (2010), and has its limitation and usefulness depending on the user's perspective (seller or buyer). However once a given model is chosen it becomes a crucial point to provide a correct and coherent approach, as we hope to provide in this paper for the case of Fisher-Weil bond duration-convexity approximation.

The results derived in this work are devoted to present the essential ideas we have launched in our previous working paper Lajili \& Rakotondratsimba (2008). They are not based on specific historical or simulated data. It means that their scope are only limited by the underlying assumptions used (as the parallel shift of the interest rate curve). For convenience, non-technical summary of the ideas underlying this present work is given in the next Section 2. Full statments of results are displayed in Section 3. We first recall in Subsections 3.1 and 3.2, the sensitivities and classical duration-convexity approximation. Then we present our contribution in Subsection 3.2. For shortness, very few numerical examples are given in Section 4 to illustrate the analytical formulas we have derived here. However the reader may consult Lajili \& Rakotondratsimba (2008) for the proofs and further details. We conclude in Section 5 .

\section{Non-technical Presentation of Our Results}

It is common in financial practice and theory to measure bond price changes, under a parallel shift of the interest rate curve, by means of sensitivities tools as the duration and convexity These last (whose the precise definitions are given in (4) and (6)) are then used by people to immunize a portfolio of assets and liabilities.

Therefore the price relative change of a bond position is seen as given approximately by the sum of two terms (see the full statement in (8)). The first term is the opposite of duration times the parallel shift value of the interest rates. The second term is the product of convexity and the square of this shift value. In the sequel, we refer such an approximation as the classical Fisher-Weil duration-convexity approximation. Actually the idea of approximation of bond change comes back to various authors as F. Macaulay (1938), F. Redington (1952)and L. Fisher and R.Weil (1971).

Such a key approximation is very often mentioned in finance text books (see for instance Hull (2009)) to be valid for small change values of the interest rates. However there is no available references which precise the exact meaning of the term small change. Moreover the accuracy of the given approximation remains also unclear. Another important deficiency of the classical duration-convexity approximation is the lack of consideration of the time-passage. Indeed with a zero shift for the interest rate, the duration-convexity approximation leads to a zero relative change for the bond. However in reality the bond value changes, though the interest rate curve remains the same due to the passage of time (see for instance the examples in Section 4). All of these three inconveniences (disregard of the time-passage, indistinctness on the shift size to use, approximation error out-of-the control) lead to a frustrating situation when using the classical Fisher-Weil approximation in the bond portfolio hedging. Apparently a rough approximation approach may lead to an economic loss which goes in the opposite of the initial hedge intention.

Our purpose in this paper is to enhance this classical duration-convexity approximation approach so as to overcome the three inconveniences mentioned above. Therefore we will show in (26) of Theorem 6 that the bond relative change may be seen as the sum of three terms. The first (deterministic) term represents the value of the passage of time not present in the classical approximation. The second term is the sum of two other terms. There is first the opposite of a modified-duration times the parallel shift value of the interest rates. The next term is the product of a modified-convexity by the square of this shift value. The third term is the remainder error term when using the 
approximation as a replacement of the initial bond change. The modified-duration and modified-convexity (see (23) and (24)) are defined similarly as the classical duration and convexity. The only change is just the incorporation of the time-passage, and the classical duration and convexity are recovered when the time passage is reduced to zero. We refer our new approximation as the modified duration-convexity approximation. In our approach, any arbitrary parallel shift of the interest rate is allowed to be used. But in return, we have to grant an attention on the size of the corresponding remainder approximation error. This last term is of course unknown, however we are able here to derive (see for instance (29) and (30)) an explicit bounds under the assumption that the shift is bounded below. This is a very minimal assumption and no hypothesis on the shift distribution is required. In other terms, we obtain a pointwise estimate of the approximation error. This is an interesting point in bond hedging for example, since a pointwise estimate leads immediately to an economical meaning (as maximum loss or gain level). In contrast the hedging error in term of variance, very often used by many authors, leaves a level of uncertainty and a lack of economical interpretation. Moreover the variance requires in priori the knowledge of the distribution associated to the interest rate shift.

\section{The Results}

\subsection{Fixed Income Value and Their Sensibilities}

In this paper we will focus on fixed income products of any kind, assuming that all future cash flows of the respective investments are known and not subject to default risk. For doing let us introduce the cash flows payment dates $t_{k}, k \in\{1, \ldots, n\}$, with $0 \leq t \leq t_{1}<\ldots<t_{k}<\ldots<t_{n}=T$. Denoting by $t$ the present time, then let us set by $\tau_{k}(t)=t_{k}-t$ the time elapsed until the $k$-th cash flow $C_{k}$ is due.

Standard par-bond products are given by the particular cash flows $C_{1}=\ldots=C_{n-1}=100 c N$ and $C_{n}=100(1+c) N$, where $100 \mathrm{~N}$ is the principal (or face value) and $c$ is the coupon rate.

No-arbitrage considerations lead us to define the price $P_{t}$ of the stream of positive cash flows $C_{1}, \ldots, C_{k}, \ldots, C_{n}$ by

$$
P_{t}=\sum_{k=1}^{n} C_{k} D\left(t, t_{k}\right)
$$

where $D\left(t, t_{k}\right)$ is a discount factor. We will work with the continuous compounding setting for which $D(t, t+\tau)$, with $0<\tau$, is given by

$$
D(t, t+\tau)=\exp \left[-r_{t, \tau} \tau\right]
$$

where $0 \leq r_{t, \tau}$. With (1) and (2), it appears that the current price $P_{t}$ depends both on the tenor $\tau_{1}(t), \ldots, \tau_{k}(t), \ldots, \tau_{n}(t)$ and the zero interest rates $r_{t, \tau_{1}(t)}, \ldots, r_{t, \tau_{k}(t)}, \ldots, r_{t, \tau_{n}(t)}$ at this time $t$. These last are defined by the interest rates curve $u \in\left[0, \infty\left[\mapsto r_{t, u}\right.\right.$.

Usually, people try to grasp the consequence of a parallel shift of the current zero interest rates by considering the quantity

$$
\sum_{k=1}^{n} C_{k} \exp \left[-\left(r_{t, \tau_{k}(t)}+\varepsilon\right) \tau_{k}(t)\right] \equiv P_{t, 0 ; \varepsilon}
$$

The concern task is then to analyze the price absolute change $P_{t, 0 ; \varepsilon}-P_{t}$ or the corresponding relative change $\frac{P_{t, 0 ; \varepsilon}-P_{t}}{P_{t}}$. The common approach of this relative change is to make use of the concept of duration and convexity.

The (Macaulay) duration, of any fixed income product whose the price is as considered in (1) and (2) is defined by

$$
\operatorname{Dur}(t)=\frac{1}{P_{t}} \sum_{k=1}^{n} \tau_{k}(t) C_{k} \exp \left[-r_{t, \tau_{k}(t)} \tau_{k}(t)\right]
$$

The duration can be interpreted as a weighted arithmetic average of the $\tau_{k}(t)$ 's with the weights $\omega_{k}(t)=\frac{1}{P_{t}} C_{k} \exp \left[-r_{t, \tau_{k}(t)} \tau_{k}(t)\right]$. As a consequence one has 


$$
\operatorname{Dur}(t) \leq(T-t) .
$$

To take into account the convexity, we need to introduce

$$
\operatorname{Conv}(t)=\frac{1}{2 P_{t}} \sum_{k=1}^{n} \tau_{k}^{2}(t) C_{k} \exp \left[-r_{t, \tau_{k}(t)} \tau_{k}(t)\right] .
$$

Similarly to (5) it can be easily seen that

$$
\operatorname{Conv}(t) \leq \frac{1}{2}(T-t)^{2} .
$$

\subsection{Classical Duration-Convexity Approximation}

From the finance literature (pioneered by the papers of F. Macaulay (1938), F. Redington (1952) and L. Fisher and R. Weil (1971) the following approximation

$$
\frac{P_{t, 0 ; \varepsilon}-P_{t}}{P_{t}} \approx-\operatorname{Dur}(t) \times \varepsilon+\operatorname{Conv}(t) \times \varepsilon^{2},
$$

is refered here as the classical (Fisher-Weil) bond duration-convexity approximation. It expresses the relationship between parallel spot rate curve shift and relative price changes of the fixed income security, and consequently plays a role in hedging risks associates to the interest rates.

In textbooks as in Hull (2009), it is often stated that (8) can be used for small values of $\varepsilon$, but there is no clear available analyses and references indicating the size of $\varepsilon$ for which it remains reasonable to make use of the approximation (8). Actually it is a consequence of the Taylor expansion for which the derivatives of order more than 3 are ignored, so that some accuracy is consequently lost. Among our contributions in this Section is to clarify the quality of such approximation (8) with respect to the size of $\varepsilon$.

Actually the strength of (8) relies on the information brought about this relative variation when the value of $\varepsilon$ is uncertain. Indeed if there is uncertainty about the value of $\varepsilon$, then $\frac{P_{t, 0 ; \varepsilon}-P_{t}}{P_{t}}$ remains also uncertain. However in most of the cases, the investor may have a more and less view about the range of values possibly taken by $\varepsilon$. For instance she/he may suspect a parallel shift of the rate curve by $\varepsilon$, with $0<\varepsilon<\varepsilon_{0}$ for some given $\varepsilon_{0}$. Therefore if the size of the remainder term related to approximation (8) is available, then we may assert that $\frac{P_{t, 0 ; \varepsilon}-P_{t}}{P_{t}} \approx-\operatorname{Dur}(t) \times \varepsilon_{1}+\operatorname{Conv}(t) \times \varepsilon_{1}^{2}$ for any suitable $\varepsilon_{1}$. The fact that $\varepsilon_{1}$ or other values is taken has few importance since in any case the real value of $\varepsilon$ at the future time cannot be determined at the present time $t$. It just gives to the investor the magnitude order of things. The main point here is rather on the error size knowledge of such an approximation. It becomes a valuable tool informing the investor on the possible economic consequences of her/his anticipation in spite of the interest rates uncertainty change.

We first state the exact value of the price relative change $\frac{P_{t, 0 ; \varepsilon}-P_{t}}{P_{t}}$.

Proposition 1: Let $\varepsilon$ with $\varepsilon \neq 0$. Then there is some real number $\rho$ such that:

$$
\begin{gathered}
\frac{P_{t, 0 ; \varepsilon}-P_{t}}{P_{t}}=-\mathrm{D} u r(t) \times \varepsilon+\operatorname{Conv}(t) \times \varepsilon^{2} \\
-\frac{1}{6 P_{t}}\left(\sum_{k=1}^{n} \tau_{k}^{3}(t) C_{k} \exp \left[-\left(r_{t, \tau_{k}(t)}+\rho\right) \tau_{k}(t)\right]\right) \varepsilon^{3} .
\end{gathered}
$$

Explicit value of $\rho$ is not known here, but the only information available we have is that $0<\rho<\varepsilon$ or $\varepsilon<\rho<0$ depending on the sign of $\varepsilon$.

The restriction to $\varepsilon \neq 0$ is considered since $P_{t, 0 ; 0}$ is trivially reduced to $P_{t}$. The identity (9) can be just obtained using the Taylor formula with remainder term written in Lagrange form (see Lajili \& Y. Rakotondratsimba (2008)for details). As a consequence the shift $\varepsilon$ has any arbitrage size, in contrast with the Fisher-Weil (8) approximation which is rather obtained from a second order Taylor expansion for which $\varepsilon$ is assumed to be infinitesimal. 
In accordance with the financial standard basic use, we limit here our analysis on the second order approximation. Though (9) may lead to an acceptable approximation of $\frac{P_{t, 0 ; \varepsilon}-P_{t}}{P_{t}}$ for small values of $\varepsilon$, it should be very insufficient when considering the absolute difference $P_{t, 0 ; \varepsilon}-P_{t}$. For instance if the precision of the relative value is about $10^{-3}$ and $P_{t}$ has a magnitude of order $10^{7}$ (as in the case of bonds with face value 100 and nominal 10000 ) then the approximation by (8) may suffer of an error of order $10^{4}$. This paper is focused on the relative change $\frac{P_{t, 0 ; \varepsilon}-P_{t}}{P_{t}}$ but for the bond hedging purpose, there is the need to consider the absolute change $P_{t, 0 ; \varepsilon}-P_{t}$ and to analyze the corresponding error approximation. The size of the estimate of this last should be acceptable in the perspective of the user and consequently a restriction on just the duration and convexity may be insufficient. Therefore introducing high order sensitivities (as can be seen in Lajili\&Rakotondratsimba (2008)) becomes useful, but here we do not pursue in such a direction.

In this Subsection we derive explicit bounds of the unknown remainder term

$$
\frac{1}{6 P_{t}} \sum_{k=1}^{n} \tau_{k}^{3} C_{k} \exp \left[-\left(r_{t, \tau_{k}(t)}+\rho\right) \tau_{k}(t)\right]
$$

Therefore for an upward shift of the interest term structure we can state the following.

Proposition 2: Let $\varepsilon>0$. Then we have

$$
\begin{aligned}
& \left|\frac{P_{t, 0 ; \varepsilon}-P_{t}}{P_{t}}-\left\{-\mathrm{D} u r(t) \times \varepsilon+\operatorname{Conv}(t) \times \varepsilon^{2}\right\}\right| \\
& \leq \frac{1}{6 P_{t}}\left(\sum_{k=1}^{n} \tau_{k}^{3}(t) C_{k} \exp \left[-r_{t, \tau_{k}(t)} \tau_{k}(t)\right]\right) \varepsilon^{3} .
\end{aligned}
$$

Observe that for $\varepsilon>0$ then $P_{t, 0 ; \varepsilon}<P_{t}$. It means that the price decreases as $\varepsilon$ increases. As in (5) and (7), it is clear that

$$
\frac{1}{P_{t}}\left(\sum_{k=1}^{n} \tau_{k}^{3}(t) C_{k} \exp \left[-r_{t, \tau_{k}(t)} \tau_{k}(t)\right]\right) \leq(T-t)^{3} .
$$

This last inequality with the second member of (10) leads to

$$
\left|\frac{P_{t, 0 ; \varepsilon}-P_{t}}{P_{t}}-\left\{-\mathrm{D} u r(t) \times \varepsilon+\operatorname{Conv}(t) \times \varepsilon^{2}\right\}\right| \leq \frac{1}{6}(T-t)^{3} \varepsilon^{3} .
$$

This estimates yields a practical quality control of the approximation of $\frac{P_{t, 0 ; \varepsilon}-P_{t}}{P_{t}}$ by $-\mathrm{D} u r(t) \times \varepsilon+\operatorname{Conv}(t) \times \varepsilon^{2}$. In other terms our estimate (12) says the following:

Corollary 3: When the spot rate curve moves in a parallel shift of size $\varepsilon$, with $0<\varepsilon$, then the error in using the classical approximation (8) does not exceed $\frac{1}{6}(T-t)^{3} \varepsilon^{3}$.

It may be noted that the maximal size error

$$
\rho_{\max }(\tau, \varepsilon) \equiv \frac{1}{6} \tau^{3} \varepsilon^{3} \quad \text { with } \tau=T-t
$$

does not depend on the bond face value. To fix the idea about the magnitude of this quantity we have the following

Table. Some values of $\rho_{\max }(\tau, \varepsilon)$ 


$\begin{array}{cccc}\varepsilon & \tau=5 \text { years } & \tau=10 \text { years } & \tau=15 \text { years } \\ 0.01 \% & 2.08 \times 10^{-11} & 1.67 \times 10^{-10} & 5.63 \times 10^{-10} \\ 0.1 \% & 2.08 \times 10^{-11} & 1.67 \times 10^{-10} & 5.63 \times 10^{-7} \\ 0.5 \% & 2.60 \times 10^{-11} & 2.08 \times 10^{-5} & 7.03 \times 10^{-5} \\ 0.75 \% & 8.78 \times 10^{-6} & 7.03 \times 10^{-5} & 2.37 \times 10^{-4} \\ 1 \% & 2.08 \times 10^{-5} & 1.67 \times 10^{-4} & 5.63 \times 10^{-5} \\ 1.5 \% & 2.08 \times 10^{-5} & 5.63 \times 10^{-4} & 1.90 \times 10^{-3} \\ 2 \% & 2.08 \times 10^{-5} & 1.33 \times 10^{-3} & 4.50 \times 10^{-3}\end{array}$

It appears here that for fixed income products whose the maturity is five years, then approximation (8) can be used up to $\varepsilon \leq 2 \%$. For a ten years maturity, it seems that the approximation is fully significant for $\varepsilon \leq 0.75 \%$. The case $0<\varepsilon$, which corresponds to a loss, deserves particular attention for the risk management point of view.

For $\varepsilon<0$, the price appreciates since $P_{t}<P_{t, 0 ; \varepsilon}$ and holding the position leads to some profit. We also would like to be able to get a similar estimate as (10) when the size of $\varepsilon$ is not too big, in the sense that

$$
|\varepsilon|=-\varepsilon<\min \left\{r_{t, \tau_{k}(t)} \mid \quad k \in\{1, \ldots, n\}\right\} .
$$

This ensures that $0<\left(r_{t, \tau_{k}}+\varepsilon\right)$ for all $k \in\{1, \ldots, n\}$. Condition (13) is consistent with the assumption that if the interest rate depreciates, then it cannot reach the zero level.

Precise statement for the analogous of Proposition 2 is as follows.

Proposition 4: Let us consider $\eta$ satisfying

$$
\eta<\min \left\{r_{t, \tau_{k}(t)} \mid \quad \text { for all } k \text { with } k \in\{1, \ldots, n\}\right\}
$$

Then for any parallel shift $\varepsilon$ satisfying

$$
-\eta \leq \varepsilon<0
$$

we have the estimates

$$
\begin{array}{r}
\left|\frac{P_{t, 0 ; \varepsilon}-P_{t}}{P_{t}}-\left\{-\operatorname{Dur}(t) \times \varepsilon+\operatorname{Conv}(t) \times \varepsilon^{2}\right\}\right| \\
\leq \frac{1}{6 P_{t}}\left(\sum_{k=1}^{n} \tau_{k}^{3}(t) C_{k} \exp \left[-\left(r_{t, \tau_{k}(t)}-\eta\right) \tau_{k}(t)\right]\right)|\varepsilon|^{3} .
\end{array}
$$

In contrast with Proposition 2, devoted to the case $\varepsilon>0$, here the estimate accuracy is only stated under condition (15), which is justified by (13).

To get a simple bound for the approximation size error as in Corollary 3, we observe that

$$
\frac{1}{P_{t}}\left(\sum_{k=1}^{n} \tau_{k}^{3}(t) C_{k} \exp \left[-\left(r_{t, \tau_{k}(t)}-\eta_{k}\right) \tau_{k}(t)\right]\right) \leq \exp [\eta(T-t)](T-t)^{3} .
$$

This last inequality with the second member of (16) leads to

$$
\left|\frac{P_{t, 0 ; \varepsilon}-P_{t}}{P_{t}}-\left\{-\mathrm{D} u r(t) \times \varepsilon+\operatorname{Conv}(t) \times \varepsilon^{2}\right\}\right| \leq \frac{1}{6} \exp [\eta(T-t)](T-t)^{3}|\varepsilon|^{3} .
$$

Corollary 5: When the spot rate curve moves in a parallel shift of size $\varepsilon$, with $\varepsilon<0$ satisfying assumptions (15) and (14), then the error in using the classical approximation (8) does not exceed $\frac{1}{6} \exp [\eta(T-t)](T-t)^{3}|\varepsilon|^{3}$.

Therefore the accuracy of approximation (8) can be quickly seen from the smallness of $\frac{1}{6} \exp [\eta(T-t)](T-t)^{3}|\varepsilon|^{3}$ when $-\eta \leq \varepsilon<0$, or $\frac{1}{6}(T-t)^{3} \varepsilon^{3}$ when $0<\varepsilon$. The values of $\rho_{\max }(\tau, \varepsilon)$ as described in the above Table may be useful to appreciate the accuracy of the error estimates. 


\subsection{Enhanced Duration-Convexity Approximation}

Let us consider again a fixed-income instrument whose the value $P_{t}$ at the current time $t$ is defined by (1) and (2). For clearness, we make a little change in our notations. Therefore the value $P_{t}$ may be considered as given by some function $P$ such that

$$
P_{t}=P\left(\tau_{1}(t), \ldots \ldots, \tau_{n}(t) ; r\left(t, \tau_{1}(t)\right), \ldots \ldots, r\left(t, \tau_{n}(t)\right)\right)
$$

where $\tau_{k}(t)=t_{k}-t$ and

$$
r\left(t, \tau_{1}(t)\right)=r_{t, \tau_{1}(t)}, \ldots \ldots, r\left(t, \tau_{n}(t)\right)=r_{t, \tau_{n}(t)}
$$

For the future time $t+s$, with $0<s$, the interest rates curve evolves to $u \mapsto r(t+s, u)$. At the current time $t$, the value of $r(t+s, u)$ is unknown and can be viewed as given by some random variable. Usually people talk about a parallel shift of the zero interest rates curve whenever for some real number $\varepsilon$

$$
r(t+s, u)=r(t, u)+\varepsilon \quad \text { for all maturitiesu } \geq 0
$$

To simplify, we assume that the elapsed time $s$ is no more than the distance between the present time $t$ and the next cash-flow payment, i.e.

$$
t<t+s \leq t_{1}<\ldots \ldots<t_{n} .
$$

This means that no cash-flow is paid until the future time $t+s$. At time $t$, the investor is willing to grasp the fixed-income future value $P_{t+s}$ which is the random variable given by:

$$
\begin{aligned}
& P_{t+s}= \\
& P\left(\tau_{1}(t+s), \ldots \ldots, \tau_{n}(t+s) ; r\left(t+s, \tau_{1}(t+s)\right), \ldots \ldots, r\left(t+s, \tau_{n}(t+s)\right)\right) .
\end{aligned}
$$

Assuming a parallel shift of the interest rates curve as in (20) and using the fact that

$$
\tau_{i}(t+s)=\tau_{i}(t)-s \quad \text { for all } i \in\{1, \ldots, n\},
$$

then $P_{t+s}$ takes the form

$$
\begin{gathered}
P\left(\tau_{1}(t)-s, \ldots \ldots, \tau_{n}(t)-s ; r\left(t, \tau_{1}(t)-s\right)+\varepsilon, \ldots \ldots, r\left(t, \tau_{n}(t)-s\right)+\varepsilon\right) \\
\equiv P_{t, s ; \varepsilon} .
\end{gathered}
$$

It should be emphasized that $P_{t, s ; \varepsilon}$ represents the fixed-income value at the future time $t+s$ when the time $t$ all the points of the zero interest rates curve is shifted by $\varepsilon$.

Let us recall that the investor main concern is to get an accurate idea (preferably at the current time $t$ ) of the difference $P_{t+s}-P_{t}$ or the corresponding relative change $\frac{P_{t+s}-P_{t}}{P_{t}}$. Therefore the main issue remains now to control the difference $P_{t, s ; \varepsilon}-P_{t}$ or the relative change $\frac{P_{t, s ; \varepsilon}-P_{t}}{P_{t}}$ whenever $s>0$. The classical approach, as we have analyzed in the previous subsection, is just focused on $P_{t, 0 ; \varepsilon}-P_{t}$ and $\frac{P_{t, 0 ; \varepsilon}-P_{t}}{P_{t}}$, i.e. just for the case $s=0$.

Here the zero interest rate curve movement is taken into consideration but the time passage is not taken into consideration. It means that the classical approach, as described in the previous Subsection, is not satisfactory the investor would like to grasp the fixed-income instrument value at a future time $t+s$ for which $s$ satisfies always $s>0$. In this paper, we would like to put in evidence that taking into account the time passage leads to enhance the approximation of the price change. Having an accurate approximation is valuable to get a good hedging and risk management. Applications of the results, we obtain here, on stress-testing and bond hedging is developed in our recent working paper Jaffal\&Rakotondratsimba (2011).

From now, we will deal with: 


$$
P_{t, s ; \varepsilon}=\sum_{k=1}^{n} C_{k} \exp \left[-\left(r_{t, \tau_{k}(t)-s}+\varepsilon\right)\left(\tau_{k}(t)-s\right)\right]
$$

which should be understood as the future price at time $t+s$ under a parallel shift $\varepsilon$ of the zero interest rates curve.

Variants of the duration and convexity introduced in (4) and (6), taking into account the passage of time, are defined by

$$
\operatorname{Dur}(t, s)=\frac{1}{P_{t, s ; 0}} \sum_{k=1}^{n}\left(\tau_{k}(t)-s\right) C_{k} \exp \left[-r_{t, \tau_{k}(t)-s}\left(\tau_{k}(t)-s\right)\right]
$$

and

$$
\operatorname{Conv}(t, s)=\frac{1}{2 P_{t, s ; 0}} \sum_{k=1}^{n}\left(\tau_{k}(t)-s\right)^{2} C_{k} \exp \left[-r_{t, \tau_{k}(t)-s}\left(\tau_{k}(t)-s\right)\right]
$$

where

$$
P_{t, s ; 0}=\sum_{j=1}^{n} C_{j} \exp \left[-r_{t, \tau_{j}(t)-s}\left(\tau_{j}(t)-s\right)\right] .
$$

Observe that $P_{t, s ; 0}$ is reduced to $P_{t}$ whenever $s$ is allowed to be equal to 0 . Similarly to (5) and (7) we also have:

$$
\operatorname{Dur}(t, s) \leq(T-(t+s)) \quad \text { and } \quad \operatorname{Conv}(t, s) \leq \frac{1}{2}(T-(t+s))^{2} .
$$

We can now state our result about the estimate of the ratio $\frac{P_{t, s ; \varepsilon}-P_{t}}{P_{t}}$ under a parallel shift of the curve of interest rates as described in (20).

Theorem 6: Let $s$, with $0<s \leq t_{1}-t$. Assume that for the future time $t+s$, the term structure of the interest rates has done a parallel shift of $\varepsilon$, as described in (20). Then for all $\varepsilon$, with $\varepsilon>0$, we have the approximation

$$
\begin{aligned}
& \frac{P_{t, s ; \varepsilon}-P_{t}}{P_{t}} \approx \frac{1}{P_{t}}\left\{\sum_{k=1}^{n} C_{k}\left(\exp \left[-r_{t, \tau_{k}(t)-s}\left(\tau_{k}(t)-s\right)\right]-\exp \left[-r_{t, \tau_{k}(t)} \tau_{k}(t)\right]\right)\right\} \\
& +\left(\frac{P_{t, s ; 0}}{P_{t}}\right)\left\{-\operatorname{Dur}(t, s) \times \varepsilon+\operatorname{Conv}(t, s) \times \varepsilon^{2}\right\} .
\end{aligned}
$$

The size of the remainder term

$$
\begin{gathered}
\operatorname{Rem}(t, s, \varepsilon) \equiv \frac{P_{t, s ; \varepsilon}-P_{t}}{P_{t}} \\
-\frac{1}{P_{t}}\left\{\sum_{k=1}^{n} C_{k}\left(\exp \left[-r_{t, \tau_{k}(t)-s}\left(\tau_{k}(t)-s\right)\right]-\exp \left[-r_{t, \tau_{k}(t)} \tau_{k}(t)\right]\right)\right\} \\
-\left(\frac{P_{t, s ; 0}}{P_{t}}\right)\left\{-\operatorname{Dur}(t, s) \times \varepsilon+\operatorname{Conv}(t, s) \times \varepsilon^{2}\right\}
\end{gathered}
$$

is given by the following estimates

$$
|\operatorname{Rem}(t, s, \varepsilon)| \leq \frac{1}{6 P_{t}}\left(\sum_{k=1}^{n}\left(\tau_{k}(t)-s\right)^{3} C_{k} \exp \left[-r_{t, \tau_{k}(t)-s}\left(\tau_{k}(t)-s\right)\right]\right) \varepsilon^{3} .
$$

As in (11) we also get : 


$$
\frac{1}{P_{t}}\left(\sum_{k=1}^{n}\left(\tau_{k}(t)-s\right)^{3} C_{k} \exp \left[-r_{t, \tau_{k}(t)-s}\left(\tau_{k}(t)-s\right)\right]\right) \leq\left(\frac{P_{t, s ; 0}}{P_{t}}\right)(T-(t+s))^{3}
$$

which, combined with (28), leads to the more practical error estimates

$$
|\operatorname{Rem}(t, s, \varepsilon)| \leq \frac{1}{6}\left(\frac{P_{t, s ; 0}}{P_{t}}\right)(T-(t+s))^{3} \varepsilon^{3} .
$$

Our result in Theorem 6 deals with the upward shift of the zero interest rates curve. For the downward movement we get the following statement.

Theorem 7: Let $s$, with $0<s \leq t_{1}-t$. Let us consider $\eta$ satisfying

$$
\eta<\min \left\{r_{t, \tau_{k}(t)-s} \mid \quad \text { for all } k \text { with } k \in\{1, \ldots, n\}\right\} .
$$

Assume that for the future time $t+s$, the term structure of the interest rates has done a parallel shift of $\varepsilon$, as described in (20). Then for all $\varepsilon$ satisfying

$$
-\eta \leq \varepsilon<0
$$

the approximation (26) remains true. The size of the remainder term is governed by the following estimates

$$
|\operatorname{Rem}(t, s, \varepsilon)| \leq \frac{1}{6 P_{t}}\left(\sum_{k=1}^{n}\left(\tau_{k}(t)-s\right)^{3} C_{k} \exp \left[-\left\{r_{t, \tau_{k}(t)-s}-\eta\right\}\left(\tau_{k}(t)-s\right)\right]\right)|\varepsilon|^{3} .
$$

Under (32) we also have

$$
|\operatorname{Rem}(t, s, \varepsilon)| \leq \frac{1}{6}\left(\frac{P_{t, s ; 0}}{P_{t}}\right) \exp [\eta(T-(t+s))](T-(t+s))^{3}|\varepsilon|^{3} .
$$

In Theorems 6 and 7, the future date $t+s$ precedes the time $t_{1}$, where the first cash flow $C_{1}$ takes place. In Lajili \& Y. Rakotondratsimba (2008) we have also considered the case where some cash-flows are paid before the considered future time $t+s$ by using the same line of ideas we have used here.

For shortness we have limited our analysis to an approximation limited to the second order. Details for high level approximation may be seen in our recent working paper Jaffal\&Rakotondratsimba (2011). The proofs of our results in Proposition 1 to Theorem 7 are also essentially contained in Lajili\&Rakotondratsimba (2008), so we do not report here the details. It may be just useful to note that Proposition 1 and the first parts of Theorem 6 and 7 are based on Taylor formula with remainder term written in Lagrange form. All the error estimates use elementary inequalities involving exponentials.

\section{Numerical Illustrations}

For our illustration, we deal with a coupon-bond with annual payment and a remaining maturity of 5 years. The face value is 100 (Euros for instance) and the coupon rate is $5 \%$. The interest rate curve is assumed to be given by the following

$$
\begin{array}{cccccc}
\text { maturity } \tau(\text { year }) & 1 & 2 & 3 & 4 & 5 \\
r(t, \tau)(\%) & 2.16 & 2.51 & 2.87 & 3.21 & 3.54
\end{array}
$$

To simplify we make use of linear interpolation to define $r(t, \tau)$ for any $\tau$ which is not among the pillars $1 y, \ldots, 5 y$. To fully appreciate the efficiency of the modified duration-convexity introduced in this paper, we consider parallel shifts of the interest rate with large sizes as

$$
\varepsilon \in\{-2 \%,-1.5 \%, \ldots, 0 \%, \ldots, 2.5 \%, 3 \%\}
$$

in coherence with the shocks we meet frequently on the market since the 2007 financial crisis. We are interested on analyzing the bond relative change after $s=30$ days and $s=90$ days. Observe that some investment manager regularly revise the allocation of their portfolio on a quarterly basis.

What we would like to put in evidence in this illustrative Section is that, our modified duration-convexity approximation fits well the bond change when compared to the classical duration-convexity approximation.

All numbers in Table1 are given in percentage. The interest rate shifts under consideration are displayed in the first 
column. In the second column we present the exact value of the bond relative change which is given by $\frac{P_{t, s ; \varepsilon}-P_{t}}{P_{t}}$, where $P_{t}$ is computed from (1) by directly using the interest rate curve mentioned above. The quantity $P_{t, s ; \varepsilon}$ is obtained by using (22) and the interpolation of the interest rate curve. In the third column of this Table we make use of the classical duration-convexity approximation as recalled in (8). The last fourth column is computed from our modified duration-convexity approximation (26).

Similarly, the result after a delay of $s=90$ days is summarized in Table2. Focusing on this last Table, we see that for $\varepsilon=0 \%$ the bond relative change is exactly $1.2272 \%$. This case shows the inappropriateness of using the classical duration-convexity, as it lacks to take into account the time passage. It is also apparent from the other cases $\varepsilon \neq 0$ that the classical approximation (described in the third column) is far from the reality (described in the second column) and should not be used. In contrast, the modified duration-convexity approach (described in the fourth column) fits well practically the bond (relative) change since the difference between the modified approximated value and the bond change varies here from -1.26 to 4.04 basis points.

Further examples and error analyses are performed in Lajili \& Rakotondratsimba (2008) and Jaffal \& Rakotondratsimba (2011). Particularly the implication of the modified duration-convexity on hedging and managing interest rate risks is developed in this last working paper.

\section{Conclusion}

The Fisher-Weil duration-convexity approximation is inaccurately formulated since the time-passage is neglected, the shift size is assumed to be infinitesimal and the error approximation is out of the control. In this paper we have presented how to enhance this classical approximation such that these simultaneous inconveniences may be overcome. Our modified approximation leads to a perfect fit of the bond change. Indeed a shift of any arbitrary size is now allowed and a deterministic and explicit estimates of the approximation error becomes available.

Though the parallel shift of the interest rate curve is a strong and unrealistic assumption it remains a standard reference among practitioners and academics. The analysis performed here finds a valuable implication in stress-testing, hedging and managing interest rate risks as recently performed in Jaffal \& Rakotondratsimba (2011). Moreover the ideas underlying the present work lead to new and practical results related to bond risk management in the framework of a one-uncertainty factor model of short interest rate, as we have been developed recently in Rakotondratsimba (2011).

\section{References}

Derman E. (2010). Metaphors, Models \& Theories. http://papers.ssrn.com/sol3/papers.cfm?abstract_id=1713405

Fisher L., \& Weil R. (1971). Coping with the risk of interest rate fluctuations: returns to bond holders from a naive and optimal strategy. Journal of Business, 44 (3), 408-31. http://dx.doi.org/10.1086/295402

Hegde S., \& Nunn K. (1988). Non infinitesimal rates changes and Macaulay duration. Journal of Portfolio Management, 14 (2), 69-73. http://dx.doi.org/10.3905/jpm.1988.409145

Hull J. (2009). Options, Futures, \& Other Derivatives. Prentice Hall, Pearson Education International.

Jaffal H., \& Rakotondratsimba Y. (2011). Enhancement of the Fisher-Weil bond technique immunization. http://papers.ssrn.com/sol3/papers.cfm?abstract_id=1970634

Jarrow R. (2010). Risk Management Models. Johnson School Research Paper Series\#38-2010. http://papers.ssrn.com/sol3/papers.cfm?abstract_id=1712086

Lajili S., \& Rakotondratsimba Y. (2008). Revisiting the bond duration-convexity approximation. http://papers.ssrn.com/sol3/papers.cfm?abstract_id=1099361

Macaulay F. (1938). The movements of interest rates, bond yields and stock prices in the United States since 1856. National Bureau of Economic Research, New York.

Nawalka S., \& Sotto G. (2009). Managing interest rate risk: the next challenge? http://papers.ssrn.com/sol3/papers.cfm?abstract_id=1392543

Rakotondratsimba Y. (2011). Interest rate sensitivities under the Vasicek and Cox-Ingersoll-Ross models.. http://papers.ssrn.com/sol3/papers.cfm?abstract_id=1977902

Redington F. (1952). Review of the principle of life-office valuations. Journal of the Institute of Actuaries 78, 316-340. 
Triana P. (2010). The flawed maths of financial models. Financial Times. http://www.ft.com/cms/s/2/2794cfc4-f97a-11df-9e29-00144feab49a.html\#axzz1hv8N871L

Table 1. Result for $\mathrm{s}=30$ days

\begin{tabular}{|l|l|l|l|}
\hline$\varepsilon$ & exact change & classical approx & modified approx. \\
\hline-2 & 9.8336 & 9.5485 & 9.8196 \\
\hline-1.5 & 7.3942 & 7.0793 & 7.3883 \\
\hline-1 & 5.0119 & 4.6648 & 5.0102 \\
\hline-0.5 & 2.6854 & 2.3050 & 2.6852 \\
\hline 0 & 0.4132 & 0 & 0.4132 \\
\hline 0.5 & -1.8059 & -2.2502 & -1.8057 \\
\hline 1 & -3.9731 & -4.4457 & -3.9714 \\
\hline 1.5 & -6.0897 & -6.5865 & -6.0841 \\
\hline 2 & -8.1569 & -8.6725 & -8.1436 \\
\hline 2.5 & -10.1759 & -10.7037 & -10.1501 \\
\hline 3 & -12.1479 & -12.6802 & -12.1034 \\
\hline
\end{tabular}

Table 2. Result for $\mathrm{s}=90$ days

\begin{tabular}{|l|l|l|l|}
\hline$\varepsilon$ & exact change & classical approx & modified approx. \\
\hline-2 & 10.3575 & 9.5485 & 10.3449 \\
\hline-1.5 & 7.9960 & 7.0793 & 7.9907 \\
\hline-1 & 5.6879 & 4.6648 & 5.6864 \\
\hline-0.5 & 3.4321 & 2.3050 & 3.4319 \\
\hline 0 & 1.2272 & 0 & 1.2272 \\
\hline 0.5 & -0.9278 & -2.2502 & -0.9276 \\
\hline 1 & -3.0341 & -4.4457 & -3.0325 \\
\hline 1.5 & -5.0928 & -6.5865 & -5.0877 \\
\hline 2 & -7.1050 & -8.6725 & -7.0929 \\
\hline 2.5 & -9.0719 & -10.7037 & -9.0484 \\
\hline 3 & -10.9944 & -12.6802 & -10.9540 \\
\hline
\end{tabular}

